\title{
El conocimiento objetivo como base para la educación según Karl R. Popper*
}

\author{
Objective knowledge as a basis for education according to \\ Karl R. Popper
}

Recibido: 08 de noviembre de 2013 - Revisado: 05 de febrero de 2014 - Aceptado: 03 de abril de 2014

Ivonne Patricia Piñeros Veloza*

\section{Resumen}

El libro con el título Conocimiento objetivo, fue publicado en 1972. En este, Popper recogió una serie de artículos donde aborda la teoría del conocimiento. Desde estos, es posible reconstruir las bases para lograr una educación crítica. El presente texto, se orienta a identificar elementos conceptuales que se relacionan con el aumento del conocimiento a partir del método del planteamiento y solución de problemas, el papel del lenguaje, el sentido de la libertad, el desarrollo del espíritu crítico y las implicaciones que estos tienen para la vida política. Lo anterior se relaciona con el proceso educativo, a fin de que los estudiantes puedan aproximarse a la verdad, apropiarse de su entorno, y plantear, cada vez más, óptimas soluciones para enfrentar los obstáculos de su diario vivir y favorecer así, el mejoramiento de la sociedad.

\section{Palabras clave}

Racionalismo crítico, conocimiento objetivo, teoría de la educación, filosofía de la educación, espíritu crítico, democracia.

\footnotetext{
Abstract

The book entitled Objective Knowledge, was published in 1972. There, Popper collects a series of articles where he deals with the theory of knowledge. From these, it is possible to reconstruct the foundation for a critical education. This text aims to identify conceptual elements that relate to the increase of knowledge from the method of approach and problem solving, the role of language, the meaning of freedom, the development of a critical spirit and their implications of for political life. This is related to the educational process, so that students can approach the truth, appropriating their environment and pose increasingly optimal solutions to face the obstacles of everyday life and thus favoring the improvement of society.

\section{Keywords}

Critical rationalism, objective knowledge, theory of education , philosophy of education, critical spirit, democracy.
}

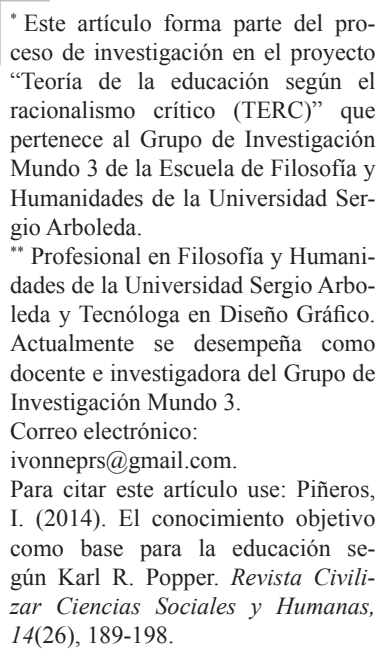


El arte de la educación o pedagogía debe ser racional a fin de que la naturaleza humana pueda desarrollarse y alcanzar su destino.

Kant

\section{Problema de investigación y método}

Desde la Escuela de Filosofía y Humanidades de la Universidad Sergio Arboleda, el Grupo de Investigación Mundo 3 avanza en el proyecto "Teoría de la educación según el racionalismo crítico (TERC)", con la intención de hacer un aporte a la comunidad académica y lograr un impacto en los procesos de enseñanza-aprendizaje y en la sociedad. Lo cual se refiere a la hipótesis del proyecto donde la educación crítica permite fortalecer la democracia. En ese sentido, el proyecto pretende explicitar cuál es la teoría educativa del racionalismo crítico (TERC) de Karl Popper, y de esta manera ofrecer soluciones para algunos problemas de la educación.

Luego de la elaboración del estado del arte, se prosigue con el análisis y la interpretación de obras principales como Lógica de la investigación cientifica, Conjeturas y refutaciones, La sociedad abierta y sus enemigos, Búsqueda sin término y, en la que enfatiza este documento, Conocimiento objetivo, en la cual es posible identificar algunas ideas y conceptos del autor que se relacionan con el proceso educativo y que permitirán aportar al desarrollo del proyecto TERC.

\section{Introducción}

Conocimiento objetivo es un libro en el que Popper recoge un conjunto de ensayos, que ayudan a entender su teoría del conocimiento o su planteamiento epistemológico según el cual, a partir de la concepción del sentido común, es posible cuestionar las teorías iniciales para construir objetivamente el conocimiento, convirtiéndolo en un proceso activo que facilita cada vez más el acercamiento a la realidad del entorno del ser humano.
Por medio de esta obra el autor aborda temas como la inducción, la evolución, el conocimiento subjetivo, la mente objetiva, la teoría de la verdad de Tarsky, entre otros que se asocian a su tesis sobre el conocimiento.

Este artículo se divide en tres partes: a) el conocimiento objetivo y el desarrollo del conocimiento; b) la idea de educación y c) el conocimiento en la sociedad. En ellas se pueden reconocer las nociones más relevantes del proceso cognitivo que permiten establecer algunas bases para la educación a partir de los planteamientos del texto en referencia.

\section{El conocimiento objetivo y el desarrollo del conocimiento}

Para comprender la concepción que Popper tiene acerca del conocimiento objetivo, es conveniente referirse a Immanuel Kant (1972), quien en su Crítica de la razón pura, trata la objetividad como la universalidad de las intuiciones que fundamentan las representaciones que dan pie para hablar del conocimiento expresado en juicios y que son en sí, relaciones objetivas que advierten sobre nuestro acercamiento a la realidad.

Stepanenko (2007) afirma que para Kant, "la objetividad de nuestros conocimientos depende del acto de juzgar, en el cual no solo articulamos conceptualmente nuestras experiencias, sino que a través de esta articulación adoptamos también una posición o adquirimos un compromiso con ese orden conceptual" (p. 69), lo que hace que el conocimiento objetivo requiera un acto de responsabilidad con los demás seres humanos a la hora de postular teorías que faciliten comprender el mundo en el que se vive.

De acuerdo con el planteamiento de Kant, Popper (1974) sostiene que el conocimiento 
humano se da a partir de la experiencia, donde es posible "tener expectativas formuladas lingüísticamente sometidas a discusión crítica" (p. 70); a ese modo de proceder, Popper lo llamará conocimiento objetivo, argumentando que, "está formado por el contenido lógico de nuestras teorías [...], las teorías publicadas en revistas y libros almacenados en bibliotecas, las discusiones de tales teorías [y] las dificultades o problemas señalados en relación con tales teorías" (p. 76), así, este tipo de conocimiento "puede aumentar y cambiar mediante la eliminación de las conjeturas lingüísticamente formuladas $[\ldots][\mathrm{e}]$ incluso puede eliminar sus propias conjeturas si es una persona autocrítica" (p. 70) con el propósito de avanzar en el conocimiento de una manera que no esté bajo influencia de vivencias personales, sino que sea el resultado de todas las posibles intervenciones que más se acerquen a la validez de una teoría. De este modo, Popper (1974) define el conocimiento objetivo como aquel que se constituye por problemas, teorías y argumentos en cuanto tales.

El conocimiento en este sentido objetivo es totalmente independiente de las pretensiones de conocimiento de un sujeto; también es independiente de su creencia o disposición de asentir o actuar. El conocimiento en sentido objetivo es conocimiento sin conocedor: es conocimiento sin sujeto cognoscente (p. 108).

Por esta razón, la subjetividad en el conocimiento se debe dejar de lado a la hora de proponer teorías; de lo contrario, no habrá ninguna forma de ampliar el conocimiento científico y, por ende, la experiencia de vida del ser humano estará cada vez más retrasada a la hora de medir sus avances sociales y científicos.

Ahora bien, por medio de la objetividad del conocimiento, su desarrollo es evidente. Popper (1974) afirma que "el método de esos diversos modos de aprender, adquirir o producir conocimiento [...] es darwinista $[\ldots]$ es un problema de selección y no de instrucción repetitiva" (p. 143); pues el conocimiento se puede desarrollar para lograr algún tipo de avance, ya que la experiencia se vuelve un factor importante, no para caer en errores pasados, sino para entenderlos y con ese punto de partida, formular nuevas hipótesis para que el conocimiento aumente, tal como lo hacen las especies que modifican sus necesidades para sobrevivir cuando su entorno cambia.

\section{La idea de educación}

Para el autor, la educación no debe ser la repetición sin sentido de experiencias ajenas, ni mucho menos la memorización de datos y fechas para que sean reproducidos a la hora del examen y olvidados luego de la prueba, sin dejar marca alguna que sea útil al momento de enfrentarse con problemas de la vida diaria. De hecho en su autobiografía intelectual, Popper (2002) reconoce que

[...] soñaba con fundar un día una escuela en la que los jóvenes pudiesen aprender sin hastío y en la que fuesen estimulados a plantear problemas y a discutirlos; una escuela en la que no hubiese que escuchar respuestas no deseadas a cuestiones no planteadas; en la que no hubiera que estudiar solo por aprobar los exámenes (p. 64).

Respecto a los procesos de aprendizaje de un estudiante, el autor afirma que el inicio de estos es el sentido común, pero si solo se enseña a través de este, es posible que los resultados no sean los mejores; pues "comenzamos [...] con un punto de partida vago y construimos sobre una base insegura" (Popper, 1974, p. 42), así será muy poco el avance del conocimiento, en este sentido, el deber de la escuela es construir, progresivamente, planteamientos sólidos. $\mathrm{Si}$ no se procede de este modo, los estudiantes se verán obligados a instruirse a la fuerza, y la institución educativa no será un lugar para aprender de la vida, sino un sitio en donde el ser humano será reconocido como una máquina de repetición y no como un individuo capaz de apropiarse de su entorno, de resolver problemas creativamente y de ser autónomo. 
La noción de educación para Popper(1974) se descubre a partir de la conceptualización de situaciones que vive cada persona para lograr -como ya se ha dicho- el conocimiento y la apropiación de su entorno. Por lo tanto, trataremos de identificar algunos conceptos que permitan una aproximación a su idea de educación, pero dado que el universo de todos los conceptos es múltiple, nos limitaremos a los que para efectos de la construcción de la TERC, nos parecen más relevantes.

\section{Sentido común y aprendizaje}

Cuando Popper (1974) habla acerca de las modos para conocer la realidad, se refiere al conocimiento del sentido común como la forma inicial para conocer el mundo. La propuesta del autor en cuanto al conocimiento, no es dejarlo como una verdad absoluta, no se construye sobre este un sistema sólido con esos principios, sino que se desarrolla una propuesta donde esos saberes puedan criticarse y someterse a contrastación en cualquier momento. Así, el filósofo de origen austriaco sostiene que "todo intento de mantener la teoría del sentido común como un todo íntegro [...] está abocado al fracaso" (p. 105), ya que la verdad está en constante reformulación a medida que se contrastan las teorías. A partir de eso, el valor de la experiencia en el aprendizaje y en el aumento del conocimiento, Popper (1974) lo argumenta de la siguiente manera:

[...] un organismo "aprende de la experiencia" si sus disposiciones reactivas cambian en el transcurso del tiempo [...]. Consideramos los procesos mediante los cuales aprende un organismo como una especie de cambio o modificación en su disposición reactiva $[\ldots]$ [estas] modificaciones [...] van a constituir el proceso de aprendizaje (p. 309).

Cuando un individuo toma consciencia de que sus fines no van a poder ser alcanzados, tendrá que sobreponerse a esa condición para superar las contrariedades y cambiar sus expec- tativas; entonces, Popper dejará claro que "el proceso de aprendizaje consiste en gran medida [...] en la eliminación de determinadas expectativas (contrariadas)" (1974, p. 310), para que el conocimiento aumente mientras que los inconvenientes se superen cada vez que sea necesario.

\section{El método}

Para Popper (1974), el avance del conocimiento y de los procesos científicos, solo es posible mediante la elección de un modelo para conocer el problema a solucionar; de esta forma, se refiere al método de la ciencia como aquel donde las conjeturas más brillantes están seguidas de inclementes intentos de refutarlas. Esto, para dejar de lado el absolutismo al que están expuestas las teorías, pues el autor considera que a través de las refutaciones, una teoría no llega a la verdad, sino que solamente se aproxima a ella.

En este sentido, para construir el conocimiento es necesario valerse del método de las conjeturas y refutaciones, pues este, además de permitir el progreso del conocimiento, hace que en el recorrido hacia la verdad se trate de buscar varias opciones, dentro de las cuales sobrevivirá la mejor, la que se aproxime a la verdad. Del mismo modo lo planteaba Darwin en cuanto a la supervivencia del más fuerte. Claro está que dicha supervivencia es válida si se posee una base crítica, para esto, Popper (1974) propone una manera de hacerlo por medio de la fórmula. $P 1 \rightarrow T T \rightarrow E E \rightarrow P 2$

Y explica:

P1 es el problema del que partimos y TT ("teoría tentativa") la primera solución imaginativa [...] EE ("eliminación de errores") consiste en el examen crítico de nuestra conjetura [...] P2 es la situación problemática tal como surge del intento crítico de resolver nuestros problemas y que lleva a un segundo intento (y así sucesivamente) (p. 157). 
Esta fórmula no solo permite tener un punto de partida claro, sino que al mismo tiempo, es factible tener una visión panorámica del problema a resolver. Entonces, al proponer múltiples soluciones, estas pueden probarse para escoger la más apropiada, con la posibilidad siempre abierta para que en otra circunstancia, al tener una nueva teoría, se pueda aplicar de nuevo la fórmula e intentar refutar la nueva proposición.

Con esto Popper (1974) explica que, para hacer razonamientos críticos es necesario acudir a un "método crítico [...] un método de ensayo y supresión de errores, de proponer teorías y someterlas a las contrastaciones más rigurosas que podamos diseñar" (p. 28), con el fin de formular una teoría más fuerte, que pueda ofrecer una mejor explicación de la realidad.

De acuerdo con lo anterior, la posibilidad de que la ciencia y el conocimiento avancen será de gran importancia, porque si vale para la ciencia, también vale para la sociedad $y$, en consecuencia, para la escuela. Por medio del ensayo y el error es viable progresar en el proceso educativo de los estudiantes y ejercitar la creatividad dentro de un grupo de personas que pretenden solucionar algún problema, ya sea de la vida diaria o de la misma institución, para aplicar la mejor medida luego de haber socializado e intentado varias alternativas.

\section{Uso del lenguaje}

En las relaciones interpersonales el elemento más valioso es el lenguaje. Para Popper (1974) es un componente imprescindible porque "nuestra humanidad, nuestra razón, se la debemos a este desarrollo [de] las funciones superiores del lenguaje, ya que nuestros poderes de razonamiento no son más que poderes de argumentación crítica" (p. 118); esas funciones superiores son la descriptiva y la argumentativa; de ellas emerge la idea reguladora de verdad, que no solo nos diferencia de los animales y las plantas, sino que facilita la apropiación y la modificación del entorno, así la descripción puede conjeturar para formular hipótesis, y la argumentación -como la más elevada de estas dos funciones-, hace posible las conversaciones críticas para que la ciencia evolucione.

En la medida en que el lenguaje sea enseñado correcta y sencillamente a los estudiantes, los resultados serán positivos para establecer comunicaciones más objetivas a la hora de abordar un problema y, por lo tanto, al momento de plantear las probables soluciones, ya que entre más sencilla sea la formulación de una teoría, es más fácil de refutar; así lo afirmaba Guillermo de Ockham cuando se refería a la simplicidad como un método para exponer teorías, "[...] entre dos teorías que diesen cuenta de un tipo de fenómenos debía preferirse la más simple [...]" (citado por Losse, 1976, p. 48). Por su parte, Popper (2002) está de acuerdo con la navaja de Ockham en el sentido en que "la teoría más simple [es] preferible, debido a su mayor poder de excluir posibles estados de cosas; o sea, por su mejor contrastabilidad" (p. 212), situación que se logra con el manejo adecuado del lenguaje para que este sirva de vehículo cuando se intenta conseguir que el conocimiento avance.

En este orden de ideas, cuando el autor se refiere a la epistemología, asegura que esta

[...] se convierte en la teoría del aumento del conocimiento. Se convierte en la teoría de la resolución de problemas o, en otras palabras, de la construcción, discusión crítica, evaluación y contrastación crítica de teorías conjeturales que compiten entre sí (Popper, 1974, p. 138).

Hechos que continuamente suceden en el aula de clase, donde el lenguaje es objetivo y mediador de situaciones; así pasa "a ser algo más que un simple medio de comunicación del que podamos prescindir [...] es más bien el medio indispensable para la discusión crítica" (p. 133) sobre la interpretación que hacemos de todos los fenómenos que se encuentran en la realidad. 
La teoría de los tres mundos, la comprensión y la solución de problemas

Cuando el conocimiento aumenta, incide en todas las esferas en las que se mueve el ser humano. En relación con esto, Popper (1974) desarrolló su teoría de los tres mundos:

[...] el primero, es el mundo físico o de los estados físicos; el segundo, es el mundo mental o de los estados mentales; el tercero, es el de los inteligibles o de las ideas en sentido objetivo, el mundo de los objetos del pensamiento posibles: el mundo de las teorías en sí mismas y sus relaciones lógicas, de los argumentos y de las situaciones problemáticas tomados en sí mismos (p. 148).

Ésta Esta tesis ayuda a comprender la realidad en la que nos movemos pues, según Popper (1974), en el conocimiento entran en juego los mundos, dado que hay interacción entre el mundo externo, la conciencia y los contenidos de las ideas.

Popper (1974) señala que "la actividad de la comprensión es esencialmente la misma que la de cualquier resolución de problemas. Es indiscutible que, como toda actividad intelectual, consta de procesos subjetivos del segundo mundo" (p. 158); es en ese mundo donde comienza el acto de conocer; primero de manera subjetiva uno empieza a cuestionarse sobre algún problema, y luego de forma objetiva es posible llegar a soluciones que pertenecerán al mundo tres: "todo análisis, intelectualmente significativo, de la actividad de comprender ha de proceder fundamentalmente, si no totalmente, mediante un análisis del manejo que hacemos de los instrumentos y unidades estructurales del tercer mundo" (p. 159), porque es en este último donde el conocimiento aumenta de manera objetiva para ser sometido a la falsación y así acercarse cada vez más a la verdad.

Cuando se llega a la claridad del problema, el proceso debe llevar a la búsqueda de una solución donde el "esquema de resolución de problemas mediante conjetura y refutación [...], puede utilizarse como una teoría explicativa de las acciones humanas, ya que podemos interpretar una acción como un intento de resolver un problema" (p. 170), que luego de haberlo experimentado en varias oportunidades y fracasando repetidas veces, puede dar pie a una solución mejor.

Así, la reparación de problemas, es el resultado del uso del principio de racionalidad, el cual, permite que el hombre sea analítico frente a determinadas situaciones, para que de modo crítico, proceda a tratar de resolver una dificultad por medio de su capacidad creativa. Aunque muchas veces los procesos conduzcan al fracaso, es necesario replantear y buscar nuevas soluciones, avanzando así en el conocimiento; un problema -afirma el autorno existe sin el conocimiento y tampoco hay conocimiento sin problemas, ya que este no se da a partir de observaciones o recolección de datos (Adorno et al., 1973).

\section{La libertad}

Además del conocimiento, la educación tiene que ver con la libertad, ella es la que ayuda a avanzar de forma crítica. Para definirla, Popper (1974) establece sus límites desde el determinismo y el indeterminismo de la siguiente manera:

[...] si el determinismo es verdadero, entonces el mundo en su conjunto es un reloj impecable que funciona con toda exactitud, lo mismo que las nubes, los organismos, los animales y los hombres. Por otro lado, si es verdadero el indeterminismo de Peirce o el de Heisenberg, o cualquier otro tipo de indeterminismo, entonces el puro azar desempeña un papel fundamental en nuestro mundo físico (p. 211).

Lo importante entonces es hallar un punto medio entre estas dos posiciones, porque no todas las decisiones se toman de modo mecánico ni de manera casual. El autor en su reflexión sobre la libertad, tiene en cuenta (en 
cierto sentido) el pensamiento del físico Arthur Comptom que se refiere al problema de "la influencia del universo de los significados abstractos sobre la conducta humana" (p. 214) y que lo soluciona cuando observa que el ser humano "[...] al desarrollar sus funciones superiores, nuestro lenguaje también ha desarrollado contenidos y significados abstractos; es decir, hemos aprendido a abstraer diversos métodos de formular o expresar teorías, así como a prestar atención a su contenido [...]" (p. 222). Popper (1974), siguiendo a Compton considera que:

[...] el control que nuestras teorías y propósitos ejercen sobre nosotros y nuestros actos es un control plástico. No nos vemos forzados a someternos al control de nuestras teorías, ya que podemos discutirlas críticamente y rechazarlas libremente si pensamos que no satisfacen nuestras normas reguladoras (pp. 222-223).

Así, no hay lugar para el dogmatismo en el conocimiento, lo que hace posible que las comunidades avancen en la manera de conocer y solucionar los problemas particulares de su propio entorno.

\section{El conocimiento en la sociedad}

Dentro de la obra revisada, Popper (1974) aborda el conocimiento en la sociedad de dos maneras: la primera que obedece a las estructuras del mundo dos, es decir, que es de índole subjetiva; y la segunda que atañe al carácter objetivo del mundo tres, advirtiendo el riesgo de las aplicaciones de las ciencias exactas para conducir tanto a seres humanos como a sociedades enteras. Aunque en Conocimiento objetivo, el autor se refiere indirectamente a la aplicación de las conjeturas y refutaciones en la sociedad, hay que tener en cuenta que sus parámetros epistemológicos son totalmente prácticos, guardan relación con la política y ocupan un lugar dentro de la vida social de las personas; la interpretación de la historia del hombre y todo lo que tiene que ver con las pro- puestas para resolver conflictos, pertenecen al mundo tres, que es de naturaleza pública, es decir, es discutible en la comunidad. En este sentido, la postura epistemológica de Popper tiene incidencia en la política, pues

La tesis de que el conocimiento puede liberarnos de la esclavitud y que podemos hacer de la idea de paz una realidad es una consecuencia del racionalismo. Lo que pienso cuando hablo de razón o racionalismo -dice Popper- [...] no es más que la convicción de que podemos aprender de nuestras faltas y errores, y en particular por medio de la crítica de otros y de la autocrítica (Burgos, 2010b, p. 78).

Este pensamiento debe considerarse y cultivarse desde los primeros años de vida y debe conservarse hasta los últimos, porque es a partir del ensayo y error como los individuos y por ende, las sociedades, evolucionan. Pues el proceso que se lleva en el conocimiento del entorno, puede incidir en la transformación de las comunidades y de las naciones en tanto que recurran a elementos críticos para solucionar, de la mejor manera, sus problemas de pobreza, analfabetismo y violencia.

\section{Conclusión}

Del estudio del texto y con los elementos descritos, es posible acercarse a la idea que Popper tiene de la educación. Si bien es cierto que no la define dentro del escrito, da algunas luces para la comprensión del fenómeno educativo y de la manera en que debe abordarse la producción del conocimiento.

La educación es una actividad que solo el hombre puede hacer con el fin de construir su historia y la de la humanidad, esto, guarda relación con la cultura y al mismo tiempo la acrecienta a través de sus expresiones ya sean artísticas o intelectuales; además, el ser humano debe tener en cuenta que esa construcción se logra a partir de la experiencia que se tiene de vivir en el mundo, la manera en que se conozca este y la forma de superar cada inconveniente. 
La escuela debe favorecer el mejoramiento de la sociedad; su compromiso es desarrollar la capacidad de formular nuevas propuestas para la solución de sus problemas; así, las generaciones venideras que sigan con esa cultura educativa podrán tomar esos avances y crear nuevas soluciones que contribuyan al progreso del conocimiento y de su sociedad. Bien sostiene Popper (1974) que "si alguien partiese de donde partió Adán no progresaría más que este" (p. 120), luego, siempre será significativo tomar las experiencias de otros para formar las propias, ya que según dirá el autor en $L a$ lógica de la investigación científica, la ciencia puede entenderse desde distintas ópticas: la epistemología, la psicología, la sociología y la política; puede ser una herramienta, un medio de producción y sirve para satisfacer las necesidades intelectuales (Popper, 1962), pero sobre todo, la ciencia tiene como objeto aproximarse a la verdad, pues esa es la manera como avanza el conocimiento científico.

Esta es, en pocas palabras, mi posición con respecto a la teoría del conocimiento. Conlleva [a] creer que el conocimiento -el conocimiento científico- es inestable, crítico, que se halla en expansión y que siempre es provisional, hipotético. Busca la verdad y trata de acercarse más a la verdad, sin llegar nunca a pretender o intentar justificar la pretensión de que la ha alcanzado (Popper, 2010, p. 51)

De acuerdo con lo precedente, al hablar de contribución a la sociedad, es importante aclarar que la educación para Popper tiene una base epistemológica sólida que va de la mano de su planteamiento sociopolítico, tal como sostiene el profesor Campo Elías Burgos (2010a): "la educación, en la medida que se promueve el espíritu crítico, contribuye al fortalecimiento de la democracia" (p. 126), dado que:

La política, la sociología y la historia son, entre otros, campos de la praxis científica; esto significa que, como en el caso de otros filósofos, en el pensamiento de Popper hay una es- trecha relación entre el planteamiento teórico -científico-y el histórico - político- (Burgos, 2004, p. 79).

Cuestión que ha venido siendo objeto de estudio por parte del Grupo de Investigación Mundo 3 de la Escuela de Filosofía y Humanidades de la Universidad Sergio Arboleda.

\section{Referencias}

Adorno, T., Popper, K., Dahrendorf, R., Habermas, J., Albert H., \& Pilot, H. (1973). La disputa del positivismo en la sociedad alemana. Barcelona, España: Ediciones Grijalbo S.A.

Antiseri, D. (2001). La Viena de Popper. Madrid, España: Unión

Burgos, C. E. (2004). La lógica de las Ciencias Sociales según Karl Popper. Bogotá, Colombia: Fondo de Publicaciones Universidad Sergio Arboleda.

Burgos, C. E. (2010a). Teoría de la Educación según el Racionalismo Crítico de Karl Popper: Bases Epistemológicas y Teoría Sociopolítica. Bogotá, Colombia: Fondo de Publicaciones Universidad Sergio Arboleda.

Burgos, C. E. (Ed). (2010b). Algunas Experiencias en Investigación Educativa. Bogotá, Colombia: Fondo de Publicaciones Universidad Sergio Arboleda.

Corvi, R. (1997). An Introduction to the Thought of Karl Popper. London: Routledge.

Darós, W. R. (1990). Realismo Crítico y Conocimiento en el Pensamiento de Karl Popper. Pensamiento, 46(182), 21-36.

Kant, I. (1972). Crítica de la Razón Pura. Ciudad de México, México: Porrúa. 
Losse, J. (1976). Introducción Histórica a la Filosofia de la Ciencia. Madrid, España: Alianza Universidad.

Magee, B. (1974). Popper. Barcelona, España: Grijalbo.

Popper, K. (1962). La Lógica de la Investigación Científica. Madrid, España: Tecnos.

Popper, K. (1974). Conocimiento Objetivo. Madrid, España: Tecnos.

Popper, K. (1979). El Desarrollo del Conocimiento Cientifico: Conjeturas y Refutaciones. Buenos Aires, Argentina: Paidós.

Popper, K. (1982). La Sociedad Abierta y sus Enemigos. España: Paidós.

Popper, K. (1984). La Miseria del Historicismo. Madrid, España: Alianza

Popper, K. (1994). Knowledge and the BodyMind Problem: in defence of interaction. Londres: Routledge.

Popper, K. (1995). En Busca de un Mundo Mejor. Barcelona: Paidós.
Popper, K. (1999). El Mundo de Parménides. Barcelona, España: Paidós.

Popper, K. (2002). Búsqueda sin Término: Una autobiografía intelectual. Madrid, España: Alianza Editorial.

Popper, K. (2010). Después de la Sociedad Abierta. Barcelona, España: Paidós.

Popper, K., \& Eccles, J. (1982). El yo y su cerebro. Barcelona: Labor.

Reale, G., \& Antiseri, D. (1995). Historia del Pensamiento Filosófico y Científico (Tomo 3). Barcelona, España: Herder.

Stepanenko, P. (2007). Una Lectura no-representacioncita de la posición de Kant Frente al Escepticismo. En F. Castañeda, V. Durán \& L. E. Hoyos (Eds.), Immanuel Kant: Vigencia de la Filosofia Crítica (pp. 67-77). Bogotá, Colombia: Siglo del Hombre Editores, Universidad Nacional de Colombia, Universidad de los Andes y Pontifica Universidad Javeriana. 